\title{
Group Replacement Strategy under Fuzzy Methods
}

\section{P. Kannagi, G. Uthra}

\begin{abstract}
Intuitionistic Fuzzy Numbers play an active role in finding an optimal solution for replacement problems under vague and uncertain situations. This paper gives a group replacement policy under fuzzy environment. Here all the costs and the number of units are taken as Triangular Intuitionistic Fuzzy Numbers (TIFNs). An example is used for illustration of the policy.
\end{abstract}

Keywords: Intuitionistic fuzzy set, Triangular Intuitionistic fuzzy Numbers, Group replacement policy.

\section{INTRODUCTION}

Fuzzy set theory wasintroduced in 1965 byZadeh[1]. Since then modifications and generalizations of the theory were developed in different directions. Intuitionistic fuzzy set is one such generalization and was introduced by P.Burillo[3] and then modified by Atanassov [5]. Fuzzy Replacement problem to determine the optimum replacement time was dealt byPranab Biswas etal [8].Replacement Problem with change in money value along with time in fuzzy environment was introduced byPranab Biswas and Surapati Pramanik [9].Intuitionistic fuzzy sets includesthe degree of hesitation along with the degree of acceptance whilethe fuzzy sets includes only the degree of acceptance. Seikh et.al [11]defined the basic arithmetic operations of generalized TIFNs and the $(\alpha, \beta)$-cut sets. G.Uthra etal. [17],[18] consideredreplacement models in intuitionistic fuzzy environment and obtained optimum results.

Replacement problems deals with items that need replacement. Items may be machines, electric bulbs or men. The need may arise due to failure, poor efficiency or breakdown. The breakdown or failure may occur suddenly or gradually. The problem is to find the best time of replacement.Replacement problems are divided into two categories:

(1)When the efficiency of the item deteriorates.

(2)When the items fail completely.

Individualreplacement policy, demands immediate replacement of the item on its failure.In Group replacement, we replace all items at a particular time whether it has failed or not. Meanwhile if any item fail we can replace it individually.

Revised Manuscript Received on December 15, 2019.

P. Kannagi, Research Scholar and Assistant Professor, Pachaiyappa's College, Chennai-600030, India

E-mail: kannagi1011@gmail,com

G. Uthra, P.G and Research Department of Mathematics, University of Madras, India. E-mail: uthragopalsamy@yahoo.com
In this paper, A Replacement problem where

During time ' $\mathrm{t}$ ', $\quad \tilde{n}_{t}$ isthe number of units failing ;

and $\tilde{n}$ is the number of units in the system ;

After time ' $\mathrm{t}$ ', $\tilde{p}(t)$ is the group replacementcost ;

On failure of an unit, $\tilde{p}_{1}$ is the cost of individual replacement ;

The replacement cost per unit in group is $\tilde{p}_{2}$ All units are assumed to be imprecise.These imprecise quantities are considered to be TIFNs.

\section{PRELIMINARIES}

Definition 2.1.Fuzzy set: Let $\mathrm{F}$ be a classical set. A fuzzy set $\tilde{F}$ is defined by $\tilde{F}=\left\{\left(f_{i}, m_{\tilde{F}}\left(f_{i}\right)\right) / f_{i} \in F\right\}$, where $m_{\tilde{F}}\left(f_{i}\right): F \rightarrow[0,1]$ is the membership function of $\tilde{F}$ and $m_{\tilde{F}}\left(f_{i}\right)$ is the degree of membership of $f_{i}$ in $\tilde{F}$.

Definition 2.2. Intuitionistic fuzzy set: Let $\mathrm{F}$ be a classical set. then an intuitionistic fuzzy set $\tilde{F}_{I}$ in $\mathrm{F}$ is given by $\tilde{F}_{I}=\left\{\left(f_{i}, m_{F_{I}}\left(f_{i}\right), \bar{m}_{F_{I}}\left(f_{i}\right)\right) / f_{i} \in F\right\}$ where $m_{F_{I}}\left(f_{i}\right): F \rightarrow[0,1] \& \bar{m}_{F_{I}}\left(f_{i}\right): F \rightarrow[0,1]$ are the membership function and the non-membership function such that $0 \leq m_{F_{I}}\left(f_{i}\right)+\bar{m}_{F_{I}}\left(f_{i}\right) \leq 1, \forall f_{i} \in F$. For $f_{i} \in F, \quad m_{F_{I}}\left(f_{i}\right)$ is thedegree of membership and $\bar{m}_{F_{I}}\left(f_{i}\right)$ is the degree of non- membership respectively.

Definition2.3. Intuitionistic Fuzzy Number: An intuitionistic fuzzy subset $\tilde{F}_{I}=\left\{\left(f_{i}, m_{F_{I}}\left(f_{i}\right), \bar{m}_{F_{I}}\left(f_{i}\right)\right) / f_{i} \in F\right\}$ of the real line $\mathrm{R}$ is called an intuitionistic fuzzy number if the following holds:

(i)There exists $\mu \in \mathrm{R}, m_{F_{I}}(\mu)=1$ and $\bar{m}_{F_{I}}(\mu)=0$. Here $\mu$ is called the mean value of $\tilde{F}_{I}$. 
(ii) $m_{F_{I}}$ is a continuous function from $\mathrm{R} \rightarrow[0,1]$ and for every element 'r'of R, $\quad 0 \leq m_{F_{I}}(r)+\bar{m}_{F_{I}}(r) \leq 1$ is true.

The membership function andthe non-membership function of $\tilde{F}_{I}$ are given by

$$
m_{F_{I}}(r)=\ldots\left\{\begin{array}{l}
0,-\infty<r \leq \mu-\alpha \\
f_{1}(r), \mu-\alpha<r<\mu \\
1, r=\mu \quad \bar{m}_{F_{I}}(r)= \\
h_{1}(r), \mu<r<\mu+\beta \\
0, \mu+\beta \leq r<\infty
\end{array}\right.
$$$$
\left\{\begin{array}{l}
1,-\infty<r \leq \mu-\alpha^{\prime} \\
f_{2}(r), \mu-\alpha^{\prime}<r<\mu \& 0 \leq f_{1}(r)+f_{2}(r) \leq 1 \\
0, r=\mu \\
h_{2}(r), \mu<r<\mu+\beta^{\prime} \& 0 \leq h_{1}(r)+h_{2}(r) \leq 1 \\
1, \mu+\beta^{\prime} \leq r<\infty
\end{array}\right.
$$

$\mu$ is the mean value of $\tilde{F}_{I}, \alpha \& \beta$ are the left $\&$ right spreads of membership function $m_{\widetilde{F}_{I}}(r)$ and $\alpha^{\prime} \& \beta^{\prime}$ represent left \& right spreads of nonmembership function $\bar{m}_{F_{I}}(r)$ respectively.

Definition2.4. : Triangular Intuitionistic Fuzzy Number: A TIFN $\tilde{a}_{1}=\left(\alpha_{1}, \beta_{1}, \gamma_{1} ; \alpha_{1}^{\prime}, \beta_{1}, \gamma_{1}^{\prime}\right) \quad$ is an intuitionistic fuzzy subset in $R$ with following $m_{\tilde{a}_{1}}(r)$ as the membership function and $\bar{m}_{\tilde{a}_{1}}(r)$ as the nonmembership function .

$m_{\tilde{a}_{1}}(r)=\left\{\begin{array}{l}\frac{r-\alpha_{1}}{\beta_{1}-\alpha_{1}} ; \alpha_{1} \leq r \leq \beta_{1} \\ \frac{\gamma_{1}-r}{\gamma_{1}-\beta_{1}} ; \beta_{1} \leq r \leq \gamma_{1} \quad \bar{m}_{\tilde{a}_{1}}(r)= \\ 0 ; \text { otherwise }\end{array}\right.$

$\left\{\begin{array}{l}\frac{\beta_{1}-r}{\beta_{1}-\alpha_{1}^{\prime}} ; \alpha_{1}^{\prime} \leq r \leq \beta_{1} \\ \frac{r-\beta_{1}}{\gamma_{1}^{\prime}-\beta_{1}} ; \beta_{1} \leq r \leq \gamma_{1}^{\prime}\end{array}\right.$

1; otherwise
Where $\alpha_{1}^{\prime} \leq \alpha_{1} \leq \beta_{1} \leq \gamma_{1} \leq \gamma_{1}^{\prime}$ and $\quad 0 \leq m_{\tilde{a}_{1}}(r) \leq 1$; $0 \leq \bar{m}_{\tilde{a}_{1}}(r) \leq 1 ; \quad 0 \leq m_{\tilde{a}_{1}}(r)+\bar{m}_{\tilde{a}}(r) \leq 1 \quad$ for $\quad$ all $r \in R$

\section{Operations on Triangular Intuitionistic Fuzzy Number:}

Let $\tilde{a}_{1}=\left(\alpha_{1}, \beta_{1}, \gamma_{1} ; \alpha_{1}^{\prime}, \beta_{1}, \gamma_{1}^{\prime}\right)$ and $\tilde{a}_{2}=$ $\left(\alpha_{2}, \beta_{2}, \gamma_{2} ; \alpha_{2}^{\prime}, \beta_{2}, \gamma_{2}^{\prime}\right)$ be two TIFNs. The arithmatic operations

Addition : $\left(\alpha_{1}, \beta_{1}, \gamma_{1} ; \alpha_{1}^{\prime}, \beta_{1}, \gamma_{1}^{\prime}\right)_{+}$

$$
\left(\alpha_{2}, \beta_{2}, \gamma_{2} ; \alpha_{2}^{\prime}, \beta_{2}, \gamma_{2}^{\prime}\right)
$$

$=$

$\left(\alpha_{1}+\alpha_{2}, \beta_{1}+\beta_{2}, \gamma_{1}+\gamma_{2} ; \alpha_{1}^{\prime}+\alpha_{2}^{\prime}, \beta_{1}+\beta_{2}, \gamma_{1}^{\prime}+\gamma_{2}^{\prime}\right)$

Subtraction: $\quad\left(\alpha_{1}, \beta_{1}, \gamma_{1} ; \alpha_{1}^{\prime}, \beta_{1}, \gamma_{1}^{\prime}\right)$.

$\left(\alpha_{2}, \beta_{2}, \gamma_{2} ; \alpha_{2}^{\prime}, \beta_{2}, \gamma_{2}^{\prime}\right)$

$=$
$\left(\alpha_{1}-\gamma_{2}, \beta_{1}-\beta_{2}, \gamma_{1}-\alpha_{2} ; \alpha_{1}^{\prime}-\gamma_{2}^{\prime}, \beta_{1}-\beta_{2}, \gamma_{1}^{\prime}-\alpha_{2}^{\prime}\right)$

Multiplication : $\left(\alpha_{1}, \beta_{1}, \gamma_{1} ; \alpha_{1}^{\prime}, \beta_{1}, \gamma_{1}^{\prime}\right) \mathrm{x}$

$\left(\alpha_{2}, \beta_{2}, \gamma_{2} ; \alpha_{2}^{\prime}, \beta_{2}, \gamma_{2}^{\prime}\right)$

$=\left(\alpha_{1} \alpha_{2}, \beta_{1} \beta_{2}, \gamma_{1} \gamma_{2} ; \alpha_{1}^{\prime} \alpha_{2}^{\prime}, \beta_{1} \beta_{2}, \gamma_{1}^{\prime} \gamma_{2}^{\prime}\right)$

Scalar Multiplication: $\mathrm{k}\left(\alpha_{1}, \beta_{1}, \gamma_{1} ; \alpha_{1}^{\prime}, \beta_{1}, \gamma_{1}^{\prime}\right)=$

$\left\{\begin{array}{l}\left(k \alpha_{1}, k \beta_{1}, k \gamma_{1} ; k \alpha_{1}^{\prime}, k \beta_{1}, k \gamma_{1}^{\prime}\right), i f k>0 \\ \left(k \gamma_{1}, k \beta_{1}, k \alpha_{1} ; k \gamma_{1}^{\prime}, k \beta_{1}, k \alpha_{1}^{\prime}\right), i f k<0\end{array}\right.$

\section{Defuzzification:}

Thedefuzzification of the TIFN $\tilde{a}_{1}=\left(\alpha_{1}, \beta_{1}, \gamma_{1} ; \alpha_{1}^{\prime}, \beta_{1}, \gamma_{1}^{\prime}\right)$ is done by the accuracy function $H\left(\tilde{a}_{1}\right)=\frac{\alpha_{1}+2 \beta_{1}+\gamma_{1}+\alpha_{1}^{\prime}+2 \beta_{1}+\gamma_{1}^{\prime}}{8}$. 


\section{GROUP REPLACEMENT POLICY UNDER INTUITIONISTIC FUZZY ENVIRONMENT}

Let us consider problem of replacement of all items at

fixed intervals $\tau$ along with replacing failed items on their failure.

LetDuring time $\tau, \tilde{n}_{t}$ be the number of units failing ; where $\tilde{n}$ is the number of unitsin the system.

After time $\tau, \tilde{p}(\tau)$ be the group replacementcost ;

On failure of an unit, $\tilde{p}_{1}$ be the cost of individual replacement ;

$\tilde{p}_{2}$ be the replacement cost per unit in group.

Then $\tilde{p}(\tau)=\tilde{p}_{1}\left[\tilde{n}_{1}+\tilde{n}_{2}+\ldots+\tilde{n}_{\tau-1}\right]+\tilde{p}_{2} \tilde{n}$

Average cost is

$\tilde{C}(\tau)=\frac{\tilde{p}(\tau)}{\tau}=\frac{\tilde{p}_{1}\left[\tilde{n}_{1}+\tilde{n}_{2}+\ldots \tilde{n}_{\tau-1}\right]+\tilde{p}_{2} \tilde{n}}{\tau}$

If $\tilde{C}(\tau)$ is minimum ' $\tau$ ' is the best age for replacement. Minimum $\widetilde{C}(\tau)$ is obtained if

$$
\begin{aligned}
& \tilde{C}(\tau+1)-\tilde{C}(\tau) \geq 0 \text { and } \tilde{C}(\tau)-\tilde{C}(\tau-1) \leq 0 . \\
& \Delta \tilde{C}(\tau)=\tilde{C}(\tau+1)-\tilde{C}(\tau)=\frac{\tilde{p}(\tau+1)}{\tau+1}-\frac{\tilde{p}(\tau)}{\tau} \\
& =\frac{\tilde{p}(\tau)+\tilde{p}_{1} \tilde{n}_{\tau}}{\tau+1}-\frac{\tilde{p}(\tau)}{\tau}=\frac{\tilde{p}_{1} \tilde{n}_{\tau}+\tilde{C}(\tau)[\tau-(\tau+1)]}{\tau(\tau+1)} \\
& =\frac{\tau \tilde{p}_{1} \tilde{n}_{\tau}-\tilde{p}(\tau)}{\tau(\tau+1)}=\frac{\tilde{p}_{1} \tilde{n}_{\tau}-\frac{\tilde{p}(\tau)}{\tau}}{\tau+1}
\end{aligned}
$$$$
\Delta \widetilde{C}(\tau)>0 \Rightarrow \tilde{p}_{1} \tilde{n}_{\tau}-\frac{\tilde{p}(\tau)}{\tau}>0
$$$$
\Rightarrow \tilde{p}_{1} \tilde{n}_{\tau}>\frac{\tilde{p}(\tau)}{\tau} \longrightarrow(1)
$$$$
\Delta \widetilde{C}(\tau-1)<0 \Rightarrow \tilde{C}(\tau)-\tilde{C}(\tau-1)<0
$$$$
\tilde{p}_{1} \tilde{n}_{\tau-1}<\frac{\tilde{p}(\tau)}{\tau} \longrightarrow(2)
$$

From (1) and (2), $\quad \tilde{p}_{1} \tilde{n}_{\tau-1}<\frac{\tilde{p}(\tau)}{\tau}<\tilde{p}_{1} \tilde{n}_{\tau}$

Thus the Group Replacement Policy is

(1) If the cost of individual replacement forperiod ' $\tau$ ' $\phi$ the average cost per period till the end of period ' $\tau$ ', then replace wholly as a group at the end ofperiod' $\tau$,

(2) If the cost of individual replacement forperiod ' $\tau$ ' $<$ the average cost per period till the end of period' $\tau$ ', then group replacement is not preferred.

\section{EXPERIMENTS AND RESULTS DESCRIPTION}

The failure rates of certain items are observed as follows:

\begin{tabular}{|l|r|r|r|r|r|}
\hline $\begin{array}{l}\text { End of } \\
\text { period }\end{array}$ & I & II & III & IV & V \\
\hline $\begin{array}{l}\text { Failure } \\
\text { probability }\end{array}$ & 0.2 & 0.3 & 0.6 & 0.85 & 1.0 \\
\hline
\end{tabular}

The cost per item of individual replacement is

$\tilde{p}_{1}=(1.20,1.25,1.30 ; 1.10,1.25,1.40) . \quad$ The decision is to be taken for simultaneousreplacement of entire items at fixed intervals and individual replacement of items on their failure. The cost of group replacement is

$\tilde{p}_{2}=(0.45,0.50,0.60 ; 0.40,0.50,0.65)$ per item, Determine the optimal interval of replacement as whole group. Also find whether individual replacement is preferable thangroup replacement.

\section{SOLUTION:}

Any item that fails during a month is replaced at the end of the month. Initially there are 100 (say) items in use .Let $\rho_{i}$ be the probability of a new item, , failing during $i^{\text {th }}$ month of its life. Thus we have

$\rho_{1}$ is 0.20

$\rho_{2}$ is $0.30-0.20=0.10$

$\rho_{3}$ is $0.60-0.30=0.30$

$\rho_{4}$ is $0.85-0.60=0.25$

$\rho_{5}$ is $1.00-0.85=0.15$ 


\section{Group Replacement Strategy under Fuzzy Methods}

Here $\rho_{1}+\rho_{2}+\rho_{3}+\rho_{4}+\rho_{5}=1$. So $\rho_{6}, \rho_{7}, \rho_{8}, \ldots$ all zero.

Let $\tilde{n}_{i} \quad$ items be replaced at $i^{\text {th }}$ month end.

$\tilde{n}$ - items at the beginning.

$\tilde{n}=\tilde{n}_{0}=(90,100,105 ; 85,100,110)$

$\tilde{n}_{1}=\tilde{n}_{0} \rho_{1}=(18,20,21 ; 17,20,22)$

$\tilde{n}_{2}=\tilde{n}_{0} \rho_{2}+\tilde{n}_{1} \rho_{1}=(12.6,14,14.7 ; 11.9,14,15.4)$

$\tilde{n}_{3}=\tilde{n}_{0} \rho_{3}+\tilde{n}_{1} \rho_{2}+\tilde{n}_{2} \rho_{1}=(31.32,34.8,36.54$ $29.58,34.8,38.28$ )

$\tilde{n}_{4}=\tilde{n}_{0} \rho_{4}+\tilde{n}_{1} \rho_{3}+\tilde{n}_{2} \rho_{2}+\tilde{n}_{3} \rho_{1}=(35.42,39.36$, $41.33 ; 33.46,39.36,43.3)$

$\tilde{n}_{5}=\tilde{n}_{0} \rho_{5}+\tilde{n}_{1} \rho_{4}+\tilde{n}_{2} \rho_{3}+\tilde{n}_{3} \rho_{2}+\tilde{n}_{4} \rho_{1}$

$$
=(31.99, \quad 35.55, \quad 37.33 ;
$$

$30.22,35.55,39.11)$

The expected life of any item $=\sum_{i=1}^{5} i \rho_{i}=3.05$

Average number of failures per month $=\frac{\tilde{n}}{3.05}=$

$(90,100,105 ; 85,100,110)$ 3.05

$=(29.51,32.79,34.43 ; 27.87,32.79,36.07)$

Therefore the Replacement cost per month

$=(29.51, \quad 32.79, \quad 34.43 ; \quad 27.87,32.79, \quad 36.07)$.

$(1.20,1.25,1.30 ; 1.10,1.25,1.40)$.

$=(35.41,40.99,44.76 ; 30.66,40.99,50.50)$

Our accuracy function $=\frac{325.29}{8}=40.66$

$\tilde{C}_{1}=(1.20,1.25,1.30 ; 1.10,1.25,1.40)$ is the cost ofindividualunit on failure.

$\tilde{C}_{2}=(0.45,0.50,0.60 ; 0.40,0.50,0.65)$ is the cost ofindividualunit in group replacement.
Average cost for different group replacement policies are:

\begin{tabular}{|c|c|c|}
\hline $\begin{array}{l}\text { End } \\
\text { of } \\
\text { mont } \\
h \text { ' } \mathrm{t} \text { ' }\end{array}$ & $\begin{array}{l}\text { Individual } \\
\text { Replacement } \\
\sum_{i=1}^{t} \tilde{n}_{i}\end{array}$ & $\begin{array}{l}\text { Group Replacement cost } \\
\tilde{p}(t)=\tilde{p}_{1}\left[\sum_{i=1}^{t} \tilde{n}_{i}\right]+\tilde{p}_{2} \tilde{n}\end{array}$ \\
\hline 1 & $(18,20,21 ; 17,20,22)$ & $\begin{array}{l}62.1,75,90.3 \\
52.7,75,102.3)\end{array}$ \\
\hline 2 & $\begin{array}{l}(30.6,34,35.7 \\
28.9,34,37.4)\end{array}$ & $\begin{array}{l}(77.22,92.5,109.41 \\
65.79,92.5,123.86)\end{array}$ \\
\hline 3 & $\begin{array}{l}\text { (61.92,68.8,72.24; } \\
58.48,68.8,75.68)\end{array}$ & $\begin{array}{l}(114.8,136,156.91 ; \\
98.33,136,177.45)\end{array}$ \\
\hline 4 & $\begin{array}{l}(97.34,108.16,113.5 \\
7 ; \\
91.94,108.16,118.98 \\
)\end{array}$ & $\begin{array}{l}(157.31,185.2,210.64 \\
135.13,185.2,238.07)\end{array}$ \\
\hline $\begin{array}{l}\text { End of } \\
\text { month } \\
\text { ' } t \text { ' }\end{array}$ & $\begin{array}{l}\text { Average Cost } \\
\frac{\tilde{p}(t)}{t}=\tilde{C}(t)\end{array}$ & $H(\widetilde{C}(t))$ \\
\hline 1 & $\begin{array}{l}(62.1,75,90.3 ; \\
52.7,75,102.3)\end{array}$ & 72.925 \\
\hline 2 & $\begin{array}{l}(38.61,46.25,54.71 \\
; 32.9,46.25,61.93)\end{array}$ & 46.644 \\
\hline 3 & $\begin{array}{l}(38.27,45.33,52.3 ; \\
32.78,45.33,59.15)\end{array}$ & 45.478 \\
\hline 4 & $\begin{array}{l}\text { ( } 39.33,46.3,52.66 ; \\
33.78,46.3,59.52)\end{array}$ & 46.311 \\
\hline \multicolumn{3}{|c|}{$H\left(\tilde{a}_{1}\right)=\frac{\alpha_{1}+2 \beta_{1}+\gamma_{1}+\alpha_{1}^{\prime}+2 \beta_{1}+\gamma_{1}^{\prime}}{8} \quad$ where } \\
\hline
\end{tabular}

Since the mean cost is minimum in the $3^{r d}$ month, group replacement should be done at the end of every $3^{\text {rd }}$ month. Also, the mean cost is $\phi 40.66$ ( the mean cost in the case of individual replacement), the individual replacement policy is preferable.

\section{CONCLUSION}

This paper provides an optimum group replacement policy under fuzzy environment. The policy is illustrated with an example, where the various parameters are TIFNs. The proposed policy is very effective in imprecise and vague situations.

\section{REFERENCES}

[1]. L.A.Zadeh (1965) Fuzzy sets, Information and Control , 8, 338-356.

[2].L.A.Zadeh (1978) Fuzzy sets as a basis for a theory of possibility, Fuzzy Sets and Systems 1, 3-28.

[3].P.Burillo ,H.Bustince and V.Mohedano ( 1994) Some definition of Intuitionistic Fuzzy Numbers, Fuzzy based.

[4].C.Y.Chiu and C.S.Park, Fuzzy cash flow analysis using present worth criterion .The Engineering Economist,39(2)(1994),113-138.

[5].K.Atanassov (1999)for FuzzyReplacement Problem Fuzzy Sets : Theory and Applications 
[6]L.A.Zadeh (2005), Toward a generalized theory of uncertainty (GTU) - An outline, Information Sciences 172, 1-40.

[7]. Yu Liu and Hong-Zhong Huang, Optimal Replacement Policy for Fuzzy Multi-State Element. J. of Multi-Valued Logic \& Soft Computing,Vol.17,pp, 69-92 (October 2009).

[8].Pranab Biswas and Surapati Pramanik, Application of Fuzzy Ranking Method to Determine the Replacement Time for FuzzyReplacement Problem . International Journal of Computer Applications (09758887) Volume 25 -No11,July 2011

[9].Pranab Biswas and Surapati Pramanik,Fuzzy Approach toReplacement Problem with Value of Money Changes Time, International Journal of Computer Application, 30(10)(2011),28-33.

[10].Kanti Swarup ,P.K.Gupta, Man Mohan: An Introduction to Management Science Operations Research (2011)

[11]. Seikh. MR, Nayak. PK, Pal M.: Generalized triangular fuzzy numbers in

intuitionistic fuzzy environment. IJERD Volume 5, 8-13 ( November 2012)

[12]. G.S.Mahapatra , T.K.Roy : Intuitionistic Fuzzy Number and Application on System Failure. Journal of Uncertain Systems Vol.7, No.2, pp 92-107, (2013)

[13].K. Pramila , G.Uthra, Optimal Solution of an Intuitionistic Fuzzy Transportation Problem, Annals of Pure and Applied Mathematics, 8 (2) (2014), 67-73.

[14].A.R.Publications, Resource Management Techniques ( O.R ) Ninth Edition (June 2015)

[15]. Abazar Kukha, Hassan Mishmast Nehi : Operations and Ranking Methods for Intuitionistic Fuzzy Numbers,IJ.Intelligent Systems and Applications, (2016), 1,35-48.

[16].S.K.Bharati: Ranking Method of Intuitionistic Fuzzy Numbers. Global Journal of Pure and Applied Mathematics, ISSN 09731768 Volume 13, Number 9 (2017), pp 4595-4608.

[17].G.Uthra, K.Thangavelu and P.Kannagi: Intuitionistic FuzzyReplacement Problem with Change in Money Value. IJMAA Volume5, Issue 4-F(2017),851-856

[18].G.Uthra, K.Thangavelu and P.Kannagi: Optimal Solution of an Intuitionistic FuzzyReplacementProblem. IJPAM Volume 119 No. 9 2018, 223-231. 